\title{
n-3 PUFA status in school children is associated with beneficial lipid profile, reduced physical activity and increased blood pressure in boys
}

\author{
Camilla T. Damsgaard ${ }^{1 *}$, Ken D. Stark ${ }^{2}$, Mads F. Hjorth ${ }^{1}$, Anja Biltoft-Jensen ${ }^{3}$, Arne Astrup ${ }^{1}$, \\ Kim F. Michaelsen ${ }^{1}$ and Lotte Lauritzen ${ }^{1}$ \\ ${ }^{1}$ Department of Nutrition, Exercise and Sports, Faculty of Science, University of Copenhagen, Frederiksberg, Denmark \\ ${ }^{2}$ Department of Kinesiology, University of Waterloo, Waterloo, Canada \\ ${ }^{3}$ Division of Nutrition, National Food Institute, Technical University of Denmark, Søborg, Denmark
}

(Submitted 20 August 2012 - Final revision received 24 January 2013 - Accepted 24 January 2013 - First published online 16 April 2013)

\begin{abstract}
Dietary $n$-3 long-chain PUFA (LC-PUFA) improve dyslipidaemia and hypertension and may affect insulin resistance and adiposity. Increasing numbers of children show signs of the metabolic syndrome (MetS), but few studies have investigated the association with $n$ - 3 LC-PUFA status. We examined the relationship between fasting whole-blood EPA or DHA (w/w\% of the total fatty acids, FA\%) and markers of the MetS (anthropometry, blood pressure, plasma lipids and glucose homeostasis) cross-sectionally in seventy-three 8-11-year-old Danish children from the OPUS School Meal Pilot Study (OPUS is an acronym of the project 'Optimal well-being, development and health for Danish children through a healthy New Nordic Diet' and is supported by a grant from the Nordea Foundation). Also, we explored the potential mediating effects of physical activity and energy intake. Girls had higher body fat percentage (BF\%), diastolic blood pressure, heart rate, plasma TAG, insulin, homeostasis model assessment-insulin resistance and glycosylated Hb than boys. Sexes did not differ in fish or macronutrient intake or whole-blood fatty acids. After adjustment for sex, age and total whole-blood fatty acid concentration, BF\% and HDL:TAG increased with whole-blood EPA $(\beta>0 \cdot 25, P<0 \cdot 05$ ), and HDL increased 0.35 (SEM $0 \cdot 13) \mathrm{mmol} / 1$ per FA\% EPA increase $(\beta=0.30$, $P=0 \cdot 008)$. Unexpectedly, DHA was positively associated with mean arterial pressure in boys $(6 \cdot 3$ (SEM $1 \cdot 7) \mathrm{mmHg} / \mathrm{FA} \% \mathrm{DHA}$ increase, $\beta=0 \cdot 62, P=0 \cdot 001$ ) and reduced physical activity in both sexes ( -44 (SEM 19) counts $/$ min per FA $\%, \beta=-0 \cdot 22, P=0 \cdot 024$ ). The associations with blood pressure and HDL remained after adjustment for physical activity, BF $\%$ and energy intake. The present study confirmed the beneficial association between $n$-3 LC-PUFA status and lipid profile seen in adults, but showed unexpected relationships with physical activity, $\mathrm{BF} \%$ and blood pressure. This is the third time we have observed such tendencies in Danish children.
\end{abstract}

Key words: $n$-3 PUFA: Fish: Metabolic syndrome

n-3 Long-chain PUFA (LC-PUFA) from fish and fish oils have been shown to improve markers of the metabolic syndrome (MetS), such as plasma TAG and blood pressure in adults and seem to reduce CHD mortality ${ }^{(1)}$. In adults, the MetS is defined as a cluster of cardiovascular risk factors including abdominal obesity, dyslipidaemia, glucose intolerance and hypertension $^{(2)}$. Although no universal definition of the MetS exists for paediatric populations, waist circumference, mean arterial pressure, plasma HDL-cholesterol and TAG, and the homeostasis model assessment-insulin resistance (HOMA-IR) index are commonly used markers of the MetS in children ${ }^{(3)}$ Metabolic dysregulations during childhood and adolescence increase the risk of the MetS and type 2 diabetes in adult$\operatorname{hood}^{(4)}$, and in parallel with the obesity epidemic, increasing numbers of children in the Western world now show features of the MetS $S^{(5)}$. However, little is known about the effects of $n$-3 LC-PUFA on MetS markers in school children.

Previous trials conducted by our group showed that fish oil supplementation reduced blood pressure in infants and teenage boys ${ }^{(6,7)}$, as is seen in adults ${ }^{(8)}$. In contrast, our previous cross-sectional study in Danish 17-year-olds, demonstrated a positive association between erythrocyte $n$ - 3 LC-PUFA status and systolic blood pressure, indicating higher blood pressure with a higher intake of $n$-3 LC-PUFA ${ }^{(9)}$. In a 7 -year followup of a randomised controlled trial, our group previously found higher blood pressure in the boys of mothers who were fish oil-supplemented, when compared with olive oilsupplemented during lactation ${ }^{(10)}$. Overall, this indicates that

\footnotetext{
Abbreviations: BF\%, body fat percentage; FA $\%, w / w \%$ of the total fatty acids in the whole blood with retention times between $12: 0$ and $22: 6 n-3 ;$ HOMA-IR, homeostasis model assessment-insulin resistance; LC-PUFA, long-chain PUFA; MetS, metabolic syndrome; OPUS, optimal well-being, development and health for Danish children through a healthy New Nordic Diet.
}

*Corresponding author: C. T. Damsgaard, fax +45 2034 8104, email ctd@life.ku.dk 
the acute effect of high-dose $n$ - 3 LC-PUFA in the form of fish oil on MetS markers may be different from that of habitual, long-term intakes of $n-3$ LC-PUFA from fish. Furthermore, studies in adults have indicated that the two major dietary $n-3$ LC-PUFA, EPA and DHA, affect different components of the MetS cluster ${ }^{(11)}$.

The boys of the fish oil-supplemented mothers also had a lower physical activity level and a higher energy intake compared with the control group ${ }^{(10)}$. Whether the effects of habitual n-3 LC-PUFA intake on MetS markers may be mediated by behavioural changes leading to, for example, changes in the physical activity level or energy intake is currently unexplained. Therefore, the aim of the present study was to investigate the association between $n-3$ LC-PUFA status, measured as EPA and DHA in the whole blood, and markers related to the MetS in 8-11-year-old Danish school children, and to explore the potential mediating effects of physical activity and energy intake.

\section{Methods}

\section{Study design and participants}

The present exploratory study was based on cross-sectional data from the OPUS (Optimal well-being, development and health for Danish children through a healthy New Nordic Diet) School Meal Pilot study. One of the main aims of the OPUS project is to investigate the effects of school meals based on the New Nordic Diet on health, well-being and cognitive performance. The present study was conducted according to the guidelines laid down in the Declaration of Helsinki and all procedures involving human subjects were approved by the Danish National Committee on Biomedical Research Ethics (no. H-1-2010-023). All children from 3rd and 4th grades ( $n$ 105) at a school in the north-eastern part of Denmark were invited to participate in the study, which was conducted from January to April 2011. Exclusion criteria were severe food intolerances or allergies or concomitant participation in other research projects involving blood sampling or radiation. Written and oral information was given before study start, and written parental consent as well as child assent was obtained for eighty-three children. The present study is based on cross-sectional baseline data from the seventy-three children from which all relevant measurements were collected.

\section{Data collection}

The participating families underwent a $2 \mathrm{~h}$ in-depth interview, either at school or in their home, about socio-economic status, food habits and other characteristics, and during which instructions on dietary recordings and physical activity and sleep measurements were given. Socio-economic status in the present study was evaluated as the reported education of the parent of the household with the highest education level and classified based on the Danish Nomenclature of Education levels obtained from Statistics Denmark 2011 (Copenhagen, Denmark). The families fell into four educational classes: (1) vocational education and basic schooling (a total of 11-13 years schooling and education); (2) short higher education (mainly theoretical, 13-15 years in total); (3) bachelor degree or equivalent (15-16 years in total); (4) master degree or higher ( $\geq 17$ years in total).

With help from their parents, the children recorded their daily intake of food and beverages for $7 \mathrm{~d}$ by a Webbased dietary assessment software developed specifically for children aged 8-11 years in the OPUS School Meal Pilot Study ${ }^{(12)}$. Amount consumed was estimated by selecting the closest portion size among four different digital images in 320 photo series. Energy and nutrient intake was calculated for each individual using the software system GIES (version $1.000 \mathrm{~d}$ - 26-02-2010) developed at the National Food Institute, Technical University of Denmark. Concomitantly, to measure physical activity, the children were asked to wear an ActiGraph ${ }^{\text {TM }}$ (GT3X + tri-axis accelerometer monitor; Actigraph) in an elastic belt tightly at the right hip for seven consecutive days, and to remove it only during water activities, i.e. showering or swimming. At the end of the observation period, data were reintegrated to $60 \mathrm{~s}$ epochs and analysed using ActiLife (version 6.0.0; ActiGraph). For the present study, only data obtained between 06.00 hours and midnight were used. Periods of at least $15 \mathrm{~min}$ of consecutive zeros using vector magnitudes were assumed to be monitor non-wear time and removed before analysis. The remaining time was considered as monitor wear time. To remove sleep and sleep activity during the evening (present as small bursts of activity), consecutive wear time of less than 60 min was disregarded before analysis. Mean physical activity was calculated as the total number of vertical counts divided by wear time and was expressed as counts/min. Time spent on sedentary activity was defined as all minutes showing $\leq 100$ counts/min, which is a widely used cut-point ${ }^{(13)}$. Furthermore, light-, moderate- and vigorous-intensity activity was defined as the number of minutes spend on physical activity of 101-2295, 2296-4011 and $\geq 4012$ counts/min, respectively, according to recently suggested paediatric cut-offs ${ }^{(13)}$. All children had a minimum of $4 \mathrm{~d}$ of at least $10 \mathrm{~h}$ of measured monitor wear time between 06.00 hours and midnight. Median days of valid recording was 7 (range 6-7) d with a mean monitor wear time (excluding sleep time) of 892 (SD 32) min/d.

Clinical measurements and blood sampling were performed in the morning in an air-conditioned, double-decker truck equipped as a mobile laboratory. Standardised procedures were used for all measurements. The children had fasted overnight except for one to two glasses of water. After 10 min rest, systolic and diastolic blood pressure and the heart rate were measured three times in the supine position with an automated device (UA-787 Plus; A\&D Medical). The mean of the last two measurements was used in the data analyses. The mean arterial pressure was calculated as: $1 / 3 \times$ systolic blood pressure $+2 / 3 \times$ diastolic blood pressure. For the measurements, two different cuff sizes were used (18-22 or $22-32 \mathrm{~cm}$ ) and the ambient temperature inside the truck was measured by an electronic thermometer. Height was measured three times to the nearest millimetre with a transportable stadiometer (CMS Weighing Equipment Limited), body weight was measured with the child in underwear or light clothes 
using a Tanita BWB-800S electronic scale (Tanita Europe) and waist circumference was measured three times to the nearest millimetre with a measuring tape at the position of the umbilicus. Body composition of the lightly clothed children was assessed by dual-energy X-ray absorptiometry using a Lunar Prodigy Pro SW scanner (GE Healthcare) with Encore software version 13.5 (Encore Software Ltd). Only whole-body fat percentage $(\mathrm{BF} \%)$ was used in the present study. The percentage of children being normal weight $v$. overweight and obese was assessed based on sex and age according to Cole et al. ${ }^{(14)}$.

\section{Blood sampling and analyses}

Local anaesthetic patches (Emla; Astra Zeneca) were sent to the families with an instruction before the clinical measurements.
A fasting venous blood sample $(35-40 \mathrm{ml})$ was drawn from the antecubital vein. Whole-blood glucose and $\mathrm{Hb}$ concentrations were analysed immediately after sampling using a Hemocue Glucose 201 and a Hemocue Hb 201 analyser, respectively (Hemocue Danmark). The Hemocue Glucose 201 calculates plasma glucose concentrations from whole blood concentrations. Glycosylated $\mathrm{Hb}$ in the whole blood was measured on a Quo-Test A1c (Quotient Diagnostics Limited). The inter-assay CV was 3.4 and $0.8 \%$ for the glucose and $\mathrm{Hb}$ analysis, respectively. According to the manufacturer, intra- and inter-assay CV were 2.6 and $1.4 \%$, respectively, for the glycosylated $\mathrm{Hb}$ analysis.

Heparinised blood was mixed with $0 \cdot 1 \%$ butylated hydroxytoluene (Sigma-Aldrich) in ethanol $(0.1 \mathrm{ml} / \mathrm{ml}$ blood) and stored at $-80^{\circ} \mathrm{C}$ for later analysis of fatty acid composition

Table 1. Characteristics of the children

(Mean values and standard deviations; medians, 25th and 75th percentiles)

\begin{tabular}{|c|c|c|c|c|c|}
\hline & \multicolumn{2}{|c|}{ Girls $(n 44)$} & \multicolumn{2}{|c|}{ Boys $(n 29)$} & \multirow[b]{2}{*}{$P^{\star}$} \\
\hline & Mean & SD & Mean & SD & \\
\hline \multicolumn{6}{|l|}{ Highest parental education (\%)† } \\
\hline Vocational education ( $11-13$ years) & \multicolumn{2}{|c|}{52} & \multicolumn{2}{|c|}{52} & 0.981 \\
\hline Short higher education (13-15 years) & \multicolumn{2}{|c|}{7} & \multicolumn{2}{|c|}{10} & \\
\hline Bachelor degree or equivalent ( $15-16$ years) & \multicolumn{2}{|c|}{25} & \multicolumn{2}{|c|}{24} & \\
\hline Master degree or higher ( $\geq 17$ years) & \multicolumn{2}{|c|}{16} & \multicolumn{2}{|c|}{14} & \\
\hline Age (years) & $10 \cdot 29$ & 0.58 & $10 \cdot 28$ & 0.58 & 0.920 \\
\hline Weight (kg) & $35 \cdot 5$ & $7 \cdot 7$ & 36.5 & $5 \cdot 2$ & 0.546 \\
\hline Height $(\mathrm{m})$ & 1.43 & 0.07 & 1.47 & 0.07 & 0.031 \\
\hline BMI $\left(\mathrm{kg} / \mathrm{m}^{2}\right)$ & $17 \cdot 2$ & 2.9 & $16 \cdot 9$ & 1.8 & 0.530 \\
\hline Weight status (normal weight/overweight + obese) (\%)† & \multicolumn{2}{|c|}{$86 \cdot 4 / 13 \cdot 6$} & \multicolumn{2}{|c|}{$93 \cdot 1 / 6 \cdot 9$} & 0.465 \\
\hline Waist (cm) & 63.3 & $8 \cdot 8$ & 63.3 & 4.9 & 0.994 \\
\hline Body fat (\%) & 24.9 & $9 \cdot 2$ & $20 \cdot 4$ & 6.5 & 0.016 \\
\hline Systolic blood pressure $(\mathrm{mmHg})$ & $107 \cdot 8$ & $7 \cdot 2$ & $107 \cdot 9$ & $7 \cdot 9$ & 0.955 \\
\hline Diastolic blood pressure $(\mathrm{mmHg})$ & $69 \cdot 0$ & 6.9 & 65.5 & 6.6 & 0.031 \\
\hline Mean arterial pressure $(\mathrm{mmHg})$ & 81.9 & $6 \cdot 1$ & $79 \cdot 6$ & 5.5 & 0.098 \\
\hline Heart rate (beats/min) & 78 & 11 & 73 & 11 & 0.034 \\
\hline Plasma glucose $(\mathrm{mmol} / \mathrm{l})$ & 5.43 & 0.40 & 5.48 & 0.40 & 0.639 \\
\hline Serum insulin $(\mathrm{pmol} / \mathrm{l})$ & 52 & 23 & 41 & 14 & 0.013 \\
\hline HOMA-IR & 1.82 & 0.84 & 1.44 & 0.58 & 0.026 \\
\hline Plasma $\mathrm{HbA} 1 \mathrm{c}(\% \mathrm{Hb})$ & 4.87 & 0.39 & 4.66 & 0.30 & 0.016 \\
\hline Plasma total cholesterol $(\mathrm{mmol} / \mathrm{l})$ & 3.94 & 0.60 & 3.79 & 0.60 & 0.322 \\
\hline Plasma LDL-cholesterol (mmol/l) & $2 \cdot 41$ & 0.46 & $2 \cdot 23$ & 0.47 & 0.111 \\
\hline Plasma HDL-cholesterol (mmol/l) & 1.22 & 0.29 & 1.31 & 0.32 & 0.183 \\
\hline \multicolumn{5}{|l|}{ Plasma TAG $\neq(\mathrm{mmol} / \mathrm{l})$} & 0.015 \\
\hline Median & \multicolumn{2}{|c|}{0.60} & \multicolumn{2}{|c|}{0.52} & \\
\hline 25th-75th percentiles & \multicolumn{2}{|c|}{$0.51-0.85$} & \multicolumn{2}{|c|}{$0.44-0.64$} & \\
\hline HDL:TAG & $2 \cdot 0$ & 0.9 & $2 \cdot 5$ & 1.0 & 0.019 \\
\hline \multicolumn{6}{|l|}{ Dietary intake } \\
\hline Energy $(\mathrm{MJ} / \mathrm{d})$ & $6 \cdot 67$ & 1.67 & 7.57 & 1.43 & 0.019 \\
\hline Protein (E\%) & 16 & 3 & 15 & 2 & 0.245 \\
\hline Carbohydrate (E\%) & 52 & 5 & 52 & 5 & 0.620 \\
\hline Fat $(\mathrm{E} \%)$ & 32 & 5 & 34 & 5 & 0.287 \\
\hline SFA (E\%) & 13 & 2 & 14 & 2 & $0 \cdot 109$ \\
\hline MUFA (E\%) & 12 & 2 & 12 & 2 & 0.804 \\
\hline PUFA (E\%) & 5 & 1 & 5 & 1 & 0.286 \\
\hline$n-6$ PUFA (E\%) & 3.8 & 0.7 & 3.7 & 0.6 & 0.256 \\
\hline$n-3$ PUFA (E\%) & 0.8 & 0.2 & 0.7 & 0.2 & 0.304 \\
\hline Fish and fish products $\ddagger(\mathrm{g} / \mathrm{d})$ & \multirow{4}{*}{\multicolumn{2}{|c|}{$\begin{array}{cc}9 \\
0-19 \\
340^{0-19}\end{array}$}} & & & 0.735 \\
\hline Median & & & \multirow{2}{*}{\multicolumn{2}{|c|}{$\begin{array}{c}12 \\
0-25\end{array}$}} & \\
\hline 25th-75th percentiles & & & & & \\
\hline Mean physical activity (counts/min) & & & 433 & 101 & $<0.001$ \\
\hline
\end{tabular}

HOMA-IR, homeostasis model assessment-insulin resistance; $\mathrm{HbA1c}$, glycosylated $\mathrm{Hb}$; E\%, percentage of energy intake.

${ }^{\star}$ Differences between the sexes were tested by the unpaired $t$ test, unless otherwise stated.

$\dagger$ The distribution within sexes was tested by the $\chi^{2}$ test.

$\ddagger$ Differences between the sexes were tested by the Mann-Whitney $U$ test. 
or was centrifuged at $2500 \mathrm{~g}, 10 \mathrm{~min}$ for storage at $-80^{\circ} \mathrm{C}$ of plasma for the measurement of cholesterol and TAG. Blood collected in serum separation tubes with gel was centrifuged at $2500 \mathrm{~g}, 10 \mathrm{~min}$ after $30 \mathrm{~min}$ coagulation at room temperature, and serum was stored at $-80^{\circ} \mathrm{C}$ for analysis of insulin concentration.

Whole-blood fatty acid composition was measured by highthroughput GC. Fatty acid methyl esters were prepared from whole blood samples by direct transesterification with convectional heat as described previously ${ }^{(15)}$. Briefly, $50 \mu$ l of whole blood were added to $1 \mathrm{ml}$ of $14 \%$ boron trifluoride in methanol (Pierce Chemicals) and $300 \mu \mathrm{l}$ of hexane containing $50 \mu \mathrm{g} / \mathrm{ml}$ of butylated hydroxytoluene (Sigma-Aldrich) and $10 \mu \mathrm{g}$ of an internal standard (22:3n-3 ethyl ester; Nu-Check Prep). Samples were briefly mixed and convectionally heated on a heating block for $60 \mathrm{~min}$ at $90^{\circ} \mathrm{C}$. Samples were allowed to cool to room temperature and additional water and hexane were added ( $1 \mathrm{ml}$ each), after which the samples were mixed for $1 \mathrm{~min}$, and then centrifuged for $5 \mathrm{~min}$ at $3000 \mathrm{rpm}$. The top hexane layer was collected for analysis on a Varian 3900 gas chromatograph (Varian Medical Systems, Inc.) equipped with a DB-FFAP $15 \mathrm{~m} \times 0.10 \mathrm{~mm}$ inner diameter $\times 0.10 \mu \mathrm{m}$ film thickness, nitroterephthalic acid-modified, polyethylene glycol, capillary column (J\&W Scientific from Agilent Technologies) with hydrogen as the carrier gas ${ }^{(16)}$. Samples $(2 \mu \mathrm{l})$ were introduced by a Varian CP-8400 autosampler (Varian Medical Systems, Inc.) into the injector heated to $250^{\circ} \mathrm{C}$ with a split ratio of $200: 1$. The initial temperature was $150^{\circ} \mathrm{C}$ with a $0.25 \mathrm{~min}$ hold followed by a $35^{\circ} \mathrm{C} / \mathrm{min}$ ramp to $200^{\circ} \mathrm{C}$, an $8^{\circ} \mathrm{C} / \mathrm{min}$ ramp to $225^{\circ} \mathrm{C}$ with a $3.2 \mathrm{~min}$ hold and then an $80^{\circ} \mathrm{C} / \mathrm{min}$ ramp up to $245^{\circ} \mathrm{C}$ with a $15 \mathrm{~min}$ hold at the end. The flame ionisation detector temperature was $300^{\circ} \mathrm{C}$ with air and $\mathrm{N}_{2}$ make-up gas flow rates of 300 and $25 \mathrm{ml} / \mathrm{min}$, respectively, and a sampling frequency of $50 \mathrm{~Hz}^{(16)}$. Total whole-blood fatty acids amounted to 175 (SD 28) $\mu \mathrm{g} / 100 \mu \mathrm{l}$ (range 112-260 $\mu \mathrm{g}$ / $100 \mu \mathrm{l})$. Numbers are evaluated in $\mathrm{w} / \mathrm{w} \%$ of the total wholeblood fatty acids with retention times between 12:0 and $22: 6 n-3$ (FA\%). The intra- and inter-assay variation of the analysis was 1.3 and $4.5 \%$ for EPA and 2.4 and $6.4 \%$ for DHA, respectively.

Plasma total and HDL-cholesterol and TAG were measured by an automated enzymatic colorimetric assay on a Vitros 5.1 FS analyser (Ortho-Clinical Diagnostics, Johnson \& Johnson). LDL-cholesterol concentrations were calculated by use of Friedewald's equation ${ }^{(17)}$. Serum insulin was measured by an automated chemiluminescent immunoassay on an ADVIA Centaur XP (Siemens Healthcare). The inter- and intra-assay variations of the analyses were 1.8 and $1.2 \%$ for total cholesterol and 1.9 and $1.2 \%$ for HDL-cholesterol, and the interassay variation was $5.9 \%$ for insulin. HOMA-IR was calculated as plasma glucose $(\mathrm{mmol} / \mathrm{l}) \times$ serum insulin $(\mathrm{mIU} / \mathrm{l}) / 22.5^{(18)}$. Insulin concentrations were converted from $\mathrm{pmol} / 1$ to $\mathrm{mIU} / 1$ by dividing with 6.945 .

\section{Statistical analysis}

Data were analysed with SPSS version 20 (IBM Corporation) and statistical significance was established at $P<0 \cdot 05$. All data are presented as means and standard deviations separately for girls and boys, as sex has a significant impact on most of the MetS variables. As the only outcome variable, plasma TAG was not normally distributed, and was log-transformed before statistical analysis. The MetS markers, whole-blood fatty acid composition and other characteristics of girls and boys were compared by the unpaired $t$ test. In order to check the validity of the measured $n-3$ LC-PUFA status, the association between whole-blood $n-3$ LC-PUFA and fish intake was tested in ANCOVA with sex as the categorical variable and age and total whole-blood fatty acid content as covariates. Investigation of the possible associations between whole-blood $n$ - 3 LC-PUFA and the MetS markers was also done in ANCOVA. For each outcome variable, two different models were used; the simple model included sex as the categorical variable and age and total whole-blood fatty acid content as covariates and for blood pressure also ambient temperature at blood sampling; the fully adjusted model further included parental education as the categorical variable as well as energy intake and mean daily physical activity and, except for anthropometric outcomes, also $\mathrm{BF} \%$ as covariates. Each model also included a sex $\times n$-3 LC-PUFA interaction term which was removed if non-significant. If this interaction term was statistically significant, subsequent analysis was performed in each of the sexes separately. The adjustment for total whole-blood fatty acid concentration to some extent adjusts for the variation in the size of plasma lipid pool (which is more likely to be influenced by the last meal) relative to the size of the more stable blood cell membrane pool. Finally, associations between EPA and DHA and either mean physical activity or energy intake were analysed by ANCOVA using simple and fully adjusted models. However, for these outcomes, energy intake, physical activity and $\mathrm{BF} \%$ were not included in the fully adjusted models, since they could be effects of the associations rather than mediators or confounders.

\section{Results}

As expected, most children were normal weight (Table 1). Girls had higher $\mathrm{BF} \%$, diastolic blood pressure, heart

Table 2. Whole-blood fatty acid composition in the children* (Mean values and standard deviations)

\begin{tabular}{|c|c|c|c|c|c|}
\hline & \multicolumn{2}{|c|}{ Girls ( $n 44)$} & \multicolumn{2}{|c|}{ Boys ( $n$ 29) } & \multirow[b]{2}{*}{$P+$} \\
\hline & Mean & SD & Mean & SD & \\
\hline SFA & $39 \cdot 6$ & 1.6 & $39 \cdot 6$ & $1 \cdot 7$ & 0.975 \\
\hline MUFA & $20 \cdot 2$ & $1 \cdot 6$ & $20 \cdot 0$ & $1 \cdot 6$ & 0.684 \\
\hline PUFA & 33.0 & $2 \cdot 1$ & 33.0 & $2 \cdot 2$ & 0.888 \\
\hline Total $n-6$ PUFA & $28 \cdot 1$ & 1.9 & $28 \cdot 2$ & 1.9 & 0.748 \\
\hline Linoleic acid $(18: 2 n-6)$ & $15 \cdot 4$ & $1 \cdot 5$ & $16 \cdot 0$ & $1 \cdot 8$ & $0 \cdot 150$ \\
\hline Arachidonic acid $(20: 4 n-6)$ & $9 \cdot 33$ & $1 \cdot 13$ & 8.98 & 1.39 & 0.244 \\
\hline Total $n$-3 PUFA & 4.96 & 0.93 & 4.75 & 0.87 & 0.318 \\
\hline$\alpha$-Linolenic acid (18:3n-3) & 0.29 & 0.07 & 0.27 & 0.08 & 0.318 \\
\hline EPA $(20: 5 n-3)$ & 0.54 & 0.25 & 0.53 & 0.27 & 0.880 \\
\hline $\mathrm{DHA}(22: 6 n-3)$ & $2 \cdot 97$ & 0.63 & 2.75 & 0.54 & 0.113 \\
\hline
\end{tabular}

${ }^{*}$ All values are given as $w / w \%$ of all whole-blood fatty acids with retention times between $12: 0$ and $22: 6 n-3$.

†Unpaired $t$ test. 
Table 3. Association between whole-blood $n-3$ long-chain PUFA (LC-PUFA) and the markers related to the metabolic syndrome*

\begin{tabular}{|c|c|c|c|c|c|c|c|c|c|c|c|c|}
\hline & \multicolumn{6}{|c|}{ EPA } & \multicolumn{6}{|c|}{ DHA } \\
\hline & \multicolumn{3}{|c|}{ Simple model† } & \multicolumn{3}{|c|}{ Fully adjusted modelł } & \multicolumn{3}{|c|}{ Simple model } & \multicolumn{3}{|c|}{ Fully adjusted model } \\
\hline & $B$ & $\beta$ & $P$ & $B$ & $\beta$ & $P$ & $B$ & $\beta$ & $P$ & $B$ & $\beta$ & $P$ \\
\hline BMI $\left(\mathrm{kg} / \mathrm{m}^{2}\right)$ & 1.87 & 0.20 & 0.114 & 2.55 & 0.27 & 0.037 & 0.62 & 0.15 & 0.223 & 0.91 & 0.22 & 0.108 \\
\hline Waist circumference $(\mathrm{cm})$ & 3.66 & 0.13 & 0.305 & 4.98 & 0.17 & 0.165 & 0.66 & 0.05 & 0.666 & 0.84 & 0.07 & 0.613 \\
\hline Body fat (\%) & 8.52 & 0.26 & 0.028 & $10 \cdot 1$ & 0.31 & 0.007 & $2 \cdot 57$ & 0.18 & 0.127 & 3.45 & 0.24 & 0.048 \\
\hline Systolic blood pressure (mmHg) & 2.76 & $0 \cdot 10$ & 0.445 & -3.48 & -0.12 & 0.336 & $\begin{array}{l}0.55 \S(F) \\
6.77(M)\end{array}$ & $\begin{array}{l}0.05(\mathrm{~F}) \\
0.46(\mathrm{M})\end{array}$ & $\begin{array}{l}0.765(\mathrm{~F}) \\
0.017(\mathrm{M})\end{array}$ & 0.93 & 0.08 & 0.571 \\
\hline Diastolic blood pressure $(\mathrm{mmHg})$ & $5 \cdot 61$ & 0.21 & 0.080 & 3.59 & $0 \cdot 13$ & 0.287 & $\begin{array}{c}-0.30 \S(\mathrm{F}) \\
6.09(\mathrm{M})\end{array}$ & $\begin{array}{r}-0.03(\mathrm{~F}) \\
0.50(\mathrm{M})\end{array}$ & $\begin{array}{l}0.862(\mathrm{~F}) \\
0.014(\mathrm{M})\end{array}$ & $\begin{array}{c}-2.26 \S(F) \\
4.41(M)\end{array}$ & $\begin{array}{r}-0.21(\mathrm{~F}) \\
0.36(\mathrm{M})\end{array}$ & $\begin{array}{l}0.273(\mathrm{~F}) \\
0.075(\mathrm{M}\end{array}$ \\
\hline Mean arterial pressure $(\mathrm{mmHg})$ & 4.66 & 0.20 & 0.095 & $1 \cdot 24$ & 0.05 & 0.663 & $\begin{array}{c}-0.02 \S(F) \\
6.32(\mathrm{M})\end{array}$ & $\begin{array}{r}-0.00(\mathrm{~F}) \\
0.62(\mathrm{M})\end{array}$ & $\begin{array}{l}0.991(\mathrm{~F}) \\
0.001(\mathrm{M})\end{array}$ & $\begin{array}{c}-1.87 \S(F) \\
4.46(\mathrm{M})\end{array}$ & $\begin{array}{r}-0.19(\mathrm{~F}) \\
0.44(\mathrm{M})\end{array}$ & $\begin{array}{l}0.291(\mathrm{~F}) \\
0.027(\mathrm{M}\end{array}$ \\
\hline HOMA-IR & 0.41 & 0.14 & 0.246 & -0.08 & -0.03 & 0.829 & 0.11 & 0.09 & 0.456 & -0.05 & -0.04 & 0.771 \\
\hline Total cholesterol $(\mathrm{mmol} / \mathrm{l})$ & 0.47 & 0.20 & 0.076 & 0.67 & 0.29 & 0.020 & $0 \cdot 10$ & 0.10 & 0.380 & 0.26 & 0.26 & 0.040 \\
\hline HDL-cholesterol (mmol/l) & 0.35 & 0.30 & 0.008 & 0.50 & 0.43 & $<0.001$ & 0.03 & 0.05 & 0.640 & 0.11 & 0.23 & 0.085 \\
\hline TAG (log $\mathrm{mmol} / \mathrm{l})$ & -0.048 & -0.08 & 0.433 & -0.08 & -0.14 & 0.246 & -0.001 & -0.00 & 0.978 & -0.005 & -0.02 & 0.839 \\
\hline HDL:TAG & 0.98 & 0.26 & 0.018 & 1.38 & 0.37 & 0.003 & 0.07 & 0.04 & 0.715 & 0.22 & 0.14 & 0.305 \\
\hline
\end{tabular}

B, slope coefficient; $\beta$, standardised regression coefficient; F, female; M, male; HOMA-IR, homeostasis model assessment-insulin resistance.

* Partial regression coefficients and $P$ values are shown based on ANCOVA models of the associations between $n-3$ LC-PUFA status and the metabolic markers. All models initially included an $n-3$ LC-PUFA $\times$ sex interaction term, which was removed from the models if non-significant.

†The simple models were adjusted for sex, age and total whole-blood fatty acid concentration and for blood pressure also ambient temperature.

†The fully adjusted models further included parental education, energy intake, mean daily physical activity and, except for anthropometric outcomes, body fat percentage.

$\S$ Due to a DHA $\times$ sex interaction $(P<0.05)$, the effect was determined separately in the sex subgroups. 
(a)

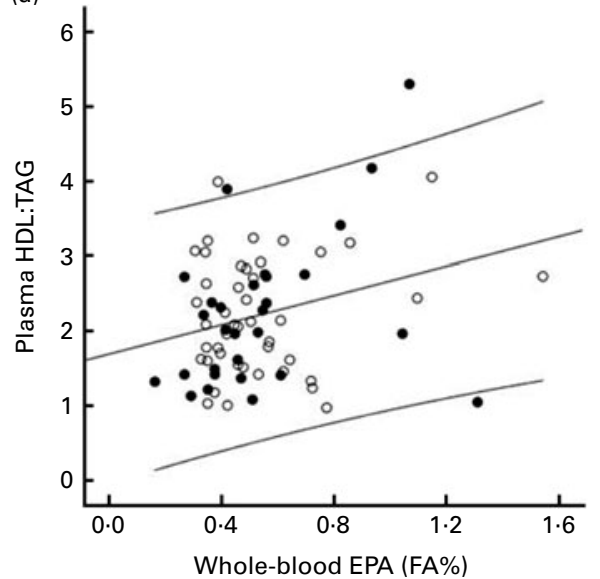

(b)

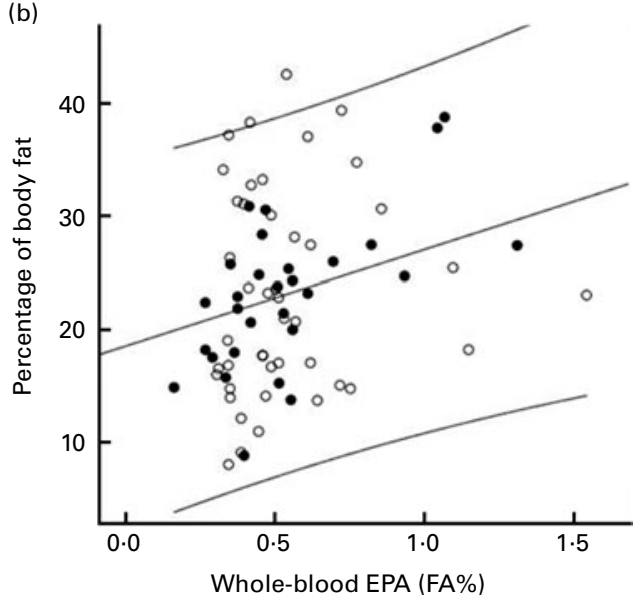

Fig. 1. (a) Plasma HDL-cholesterol:TAG and (b) body fat percentage $v$. whole-blood EPA status. Variables on both axes were adjusted for sex, age and total whole-blood fatty acid concentration (simple model). Regression lines and $95 \% \mathrm{Cl}$ are shown ( $\bullet$, boys; $\mathrm{O}$, girls), (a) $\beta=0.28, P=0.015$ and (b) $\beta=0.26$, $P=0.025$. FA\%, w/w\% of the total fatty acids in the whole blood.

rate, serum insulin, HOMA-IR, glycosylated $\mathrm{Hb}$, TAG and HDL:TAG, and were less physically active than boys. Girls and boys spent 509 (SD 68) v. 497 (SD 49) $\mathrm{min} / \mathrm{d}(P=0.43)$ on sedentary activity, $354(\mathrm{SD} \quad 61) \quad v .348(\mathrm{SD} \quad 43) \mathrm{min} / \mathrm{d}$ $(P=0.65)$ on light activity, 21 (SD 10) v. 38 (SD 14) $\mathrm{min} / \mathrm{d}$ $(P>0.001)$ on moderate activity and 7 (SD 5) and 11 $(\mathrm{SD} 8) \mathrm{min} / \mathrm{d}(P=0.009)$ on vigorous activity, respectively. We found no differences in the intake of macronutrients or fish between the sexes (Table 1).

No differences in whole-blood fatty acid composition were seen between the sexes (Table 2). Whole-blood EPA and DHA were both associated with fish intake adjusted for age, sex and total whole-blood fatty acid concentration $(\beta=0 \cdot 30$, $P=0.011$ for EPA and $\beta=0.28, P=0.017$ for DHA). We also assessed other potential composite whole-blood markers of fish intake: the $n-6: n-3$ PUFA ratio, $n-3$ highly unsaturated fatty acid:total highly unsaturated fatty acid and DHA:total PUFA, which were all found to be associated with fish intake ( $\beta$ values about 0.35 and $P$ values about 0.002 ).

Whole-blood EPA was positively associated with $\mathrm{BF} \%$, also after adjustment for energy intake and physical activity (Table 3 and Fig. 1), whereas an association between wholeblood EPA and BMI was found only in the fully adjusted model. BF\% was also positively associated with DHA in the fully adjusted model, but the association was much weaker than that for EPA (Table 3). We found higher HDL-cholesterol and HDL:TAG with increasing EPA in the whole blood (Fig. 1), and these associations were strengthened in the fully adjusted models (Table 3). Total plasma cholesterol was positively associated with both EPA and DHA in the fully adjusted models, but no association was seen for LDL-cholesterol (data not shown). Neither blood glucose, insulin, HOMA-IR nor glycosylated $\mathrm{Hb}$ was associated with EPA or DHA.

When we used systolic, diastolic and mean arterial blood pressure as the outcomes, we found a significant interaction between whole-blood DHA and sex $(P=0.041,0.027$ and 0.010 , respectively; Table 3 ). Therefore, we performed the simple analyses for blood pressure separately in the sexes.
These revealed positive associations between whole-blood DHA and both systolic and diastolic blood pressure in boys only, and most highly significant for mean arterial pressure (Table 3 and Fig. 2). The interaction between DHA and sex was also found in the fully adjusted models, but only borderline significant for systolic blood pressure $(P=0 \cdot 052$; Table 3$)$. Despite the interaction, DHA was not associated with diastolic blood pressure in boys or girls separately in the fully adjusted models. Heart rate was not associated with EPA or DHA in either the simple or the fully adjusted model $(P>0 \cdot 32)$. Systolic, diastolic and mean arterial blood pressure, adjusted for age and sex, were all strongly associated with $\mathrm{BF} \%(P<0 \cdot 005)$.

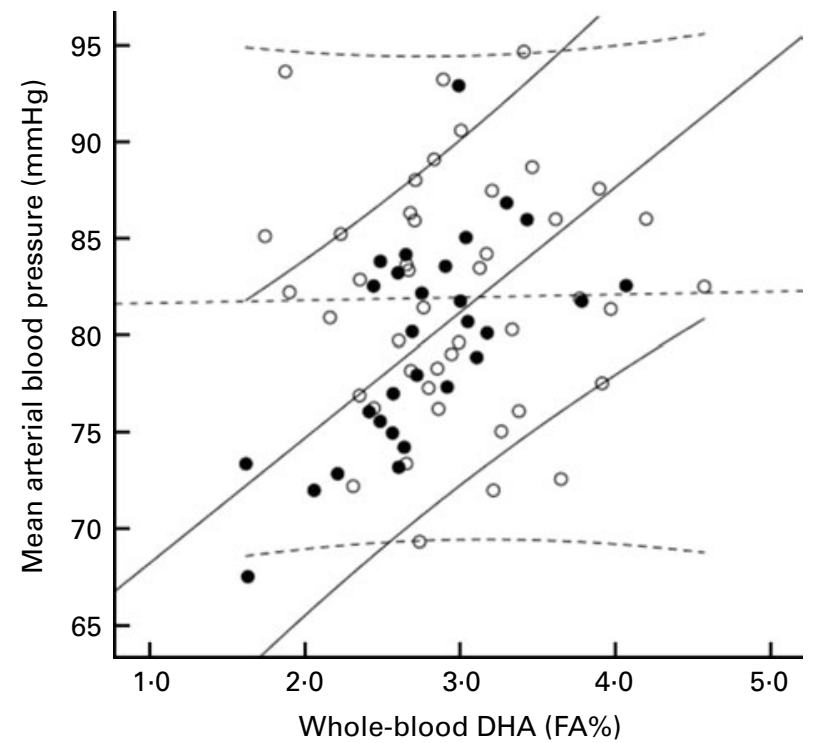

Fig. 2. Mean arterial blood pressure increases with increasing whole-blood DHA status in boys only. Variables on both axes were adjusted for age, total whole-blood fatty acid concentration and ambient temperature (simple model). Regression lines and $95 \% \mathrm{Cl}$ are given ( $\_$and $\bullet$, boys: $\beta=0.64$, $P<0.001$; $O$ and.... , girls: $\beta=0.02, P=0.92)$. FA\%, w/w\% of the total fatty acids in the whole blood. 


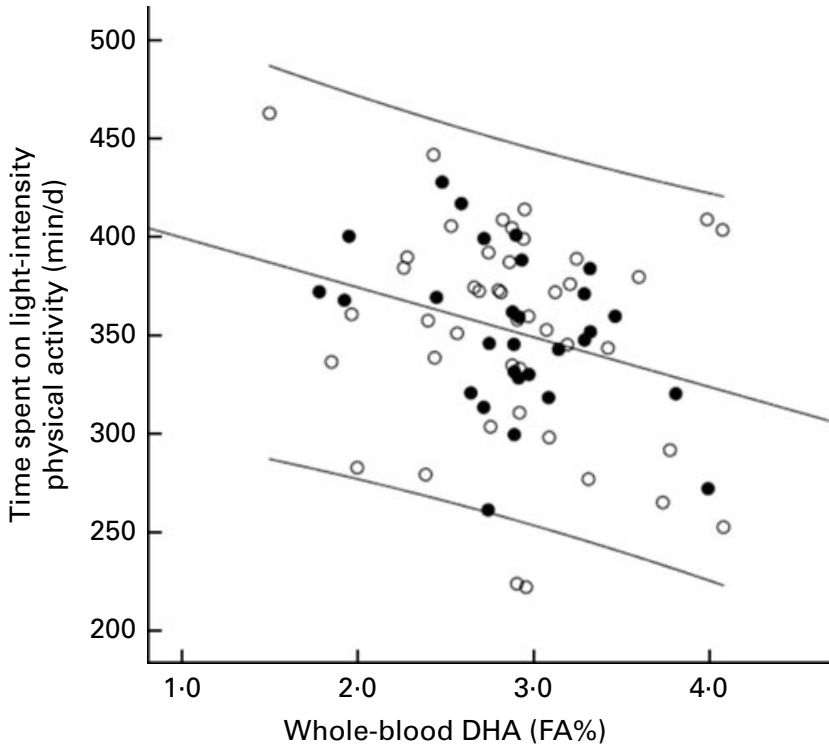

Fig. 3. Time spent on light-intensity physical activity decreases with increasing whole-blood DHA. Light physical activity was defined as 101-2295 counts/min. Variables on both axes were adjusted for sex, age, parental education and total whole-blood fatty acid concentration (simple model). Regression lines and $95 \% \mathrm{Cl}$ are given ( $\bullet$, boys; $\bigcirc$, girls), $\beta=-0.28$ $P=0.019$. $F A \%, w / w \%$ of the total fatty acids in the whole blood.

No association between either EPA or DHA and energy intake was seen in either the simple or fully adjusted models (data not shown). The mean physical activity was negatively associated with whole-blood DHA in the fully adjusted model $(B=-44$ (SEM 19$)$ counts $/$ min per $\mathrm{FA} \%, \beta=-0 \cdot 22$, $P=0.024)$ including parental education, but only borderline significant in the simple model $(\beta=-0 \cdot 17, P=0 \cdot 086)$. When we separated physical activity into time spent on sedentary, light, moderate and vigorous activity, DHA was associated with time spent on light physical activity only $(B=-25$ (SEM 11) $\mathrm{min} / \mathrm{d}$ per FA\%, $\beta=-0.28, \quad P=0.024$ ) (Fig. 3). Whole-blood EPA was not associated with physical activity in any of the models (data not shown).

\section{Discussion}

Few studies have investigated the potential associations between $n$ - 3 LC-PUFA status and markers of the MetS in children. In the present study, we consistently found higher plasma HDL-cholesterol and HDL:TAG with higher wholeblood EPA in 8-11-year-old Danish school children, despite an increased $\mathrm{BF} \%$ with higher EPA status. Most trials in adults have shown TAG-lowering effects of fish and $n$ - 3 LC-PUFA over a wide range of intakes ${ }^{(1)}$. Although some studies have seen increased HDL-cholesterol and total cholesterol with fish oil supplementation, meta-analyses have shown that these effects are negligible in adults ${ }^{(19)}$. Evidence regarding the relationship between $n$-3 LC-PUFA intake or status and plasma lipid profile in children is sparse. In line with the present findings, we previously found increased plasma HDL, but no effect on TAG in slightly overweight teenage boys randomised to fish oil-enriched breads ${ }^{(7)}$. In infants, previous work from our group showed increases in LDL-cholesterol and total cholesterol, but not HDL-cholesterol with fish oil supplementation and a negative association between $n$-3 LC-PUFA status and $\mathrm{TAG}^{(6)}$. In our previous cross-sectional study among 17-year-old Danes, there was no association between $n$-3 LC-PUFA status and plasma lipid profile ${ }^{(9)}$; however, in contrast to the present study, $n$-3 LC-PUFA status was measured in erythrocytes in that study. Mechanistically, the effect of $n$-3 LC-PUFA on plasma lipid profile is believed to be mediated by the modulation of transcription factors that are regulated by the liver $\mathrm{X}$ receptor- $\alpha /$ retinoid $\mathrm{X}$ receptor- $\alpha$ heterodimer and which control TAG assembly and oxidation in the liver ${ }^{(20)}$. These receptors have counteracting effects on reverse cholesterol transport and thereby modulate plasma HDL-cholesterol levels ${ }^{(20)}$

To our surprise, we found that mean arterial blood pressure was increased with higher DHA status among boys in the present study, and the same overall pattern was seen for diastolic blood pressure. This is in contrast with the blood pressurelowering effect of fish oil supplementation, which has been demonstrated in adults ${ }^{(8)}$ and in our previous randomised trials among children of different ages ${ }^{(6,7)}$. A follow-up of children who received $n$ - 3 LC-PUFA during the first 4 months of life also showed reduced blood pressure 6 years later in the fish oil-supplemented group when compared with the control $^{(21)}$. However, the findings of the present study are in agreement with our recent study in 17 -year-olds in which mean arterial pressure and diastolic blood pressure were positively associated with $n-3$ LC-PUFA status ${ }^{(9)}$. Also, we previously found similar blood pressure-increasing effects in the 7-year-olds who were exposed to a high intake of $n-3$ LC-PUFA early in life via the breast milk of their fish oilsupplemented mothers - especially among boys ${ }^{(10)}$.

Apart from the blood pressure effect, the physical activity of children was reduced with higher DHA status in both sexes in the present study, particularly physical activity of light intensity. Our previous study showed a lower physical activity level in children whose mothers received fish oil during lactation, again mostly among boys ${ }^{(10)}$. Furthermore, it has recently been shown that a high dose of $n-3$ PUFA lowered locomotor activity in mice ${ }^{(22)}$. Obesity and body fat are associated with increased blood pressure in children ${ }^{(23)}$ and physical activity is known to reduce blood pressure in adults ${ }^{(24)}$. The increased $\mathrm{BF} \%$ and reduced physical activity with increasing $n$-3 LC-PUFA status in the present study may be partial mediators of the associations between DHA and blood pressure in boys, since these were weakened by adjustment for $\mathrm{BF} \%$ and physical activity. Behavioural mechanisms and central nervous system-related effects may be in play. Lightintensity physical activity covers a broad spectrum of activities with an intensity between sedentary activities, such as lying and sitting, and more demanding activities such as brisk walking and bicycling ${ }^{(13,25)}$. We speculate that higher $n-3$ LC-PUFA intake and status make the children calmer and that they therefore spent less time on these types of activities. In animals, $n-3$ LC-PUFA have been shown to modulate noradrenaline secretion and serotonin and dopamine systems ${ }^{(26,27)}$, which are involved in the regulation of mood, blood pressure and heart rhythm. Also, trials among students 
and substance abusers have shown that fish oil lowers anxiety and aggression ${ }^{(28,29)}$. The blood pressure-increasing effects of higher habitual DHA status could be indirectly mediated via a reduction in physical activity. In contrast, the opposite and direct blood pressure-lowering effects of high-dose $n-3$ LC-PUFA supplementation, for example in the form of fish oil, may overrule the potential behaviour-mediated blood pressure-increasing association with habitual DHA status. However, these considerations are highly speculative and should be investigated further. Causality cannot be determined from the present explorative cross-sectional study and whether the lower physical activity and higher BF\% and mean arterial blood pressure with increasing $n$-3 LC-PUFA status have negative implications for long-term cardiovascular risk is unknown. Although the Nordic countries may have a higher fish intake and thereby higher $n$-3 LC-PUFA consumption than many other Western populations, our dietary data showed that fish consumption in this group of school children was only about one-third of the recommended $200-300 \mathrm{~g} /$ week in the Nordic countries ${ }^{(30)}$, and potentially suboptimal. Fish intake was comparable with that reported in a national sample of Danish children ${ }^{(31)}$.

The present study used whole-blood EPA and DHA as markers of $n$-3 LC-PUFA status, and they were shown to reflect the reported fish intake of children. In a small sample of normolipidaemic individuals, erythrocytes, plasma and buffy coat (containing mainly leucocytes) accounted for approximately $30-35,50-60$ and $10-15 \%$ of the total fatty acids in the whole blood (K. D. Stark and A. H. Metherel, unpublished results). Whereas erythrocyte $n$-3 LC-PUFA reflect intakes over the last months, plasma $n-3$ LC-PUFA reflect the last days or weeks; the response of the whole blood to dietary $n-3$ LC-PUFA supplementation has been shown to be intermediate between plasma and erythrocytes ${ }^{(32)}$. Although erythrocyte $n$-3 LC-PUFA would reflect even longer-term intakes, whole blood was therefore still a relevant analysis material and has the benefit that elaborate erythrocyte washing, which would have been difficult in the laboratory truck, is not required.

As discussed, EPA was correlated mainly with the plasma lipid profile and DHA with blood pressure and physical activity. In a recent literature review, Mozaffarian \& Wu ${ }^{(33)}$ concluded that the clinical effects of EPA and DHA seem to differ on exactly HDL-cholesterol and blood pressure. However, in a meta-analysis, DHA turned out also to be the most efficient with respect to the modulation of the plasma lipid profile $^{(11)}$. We do not know whether the different associations found in the present study reflect that (1) the effects are mediated via different molecular mechanisms with specific sensitivity to EPA and DHA or (2) EPA and DHA are merely markers of different pools of $n$ - 3 LC-PUFA in the whole blood. DHA tends to be mainly located in inner membranes of lipoproteins and erythrocytes, whereas EPA tends to be mainly found in the more dynamic outer membranes ${ }^{(32)}$. In line with this, plasma EPA has been shown to respond more readily to the immediate intake of $n$-3 PUFA when compared with $\mathrm{DHA}^{(34)}$. Therefore, mechanism (2) described above is certainly a possibility. Also, it seems plausible that the plasma lipid profile is affected by the acute intake via modulation of liver receptors, whereas the effects on blood pressure and behaviour would require a more stable supply of $n$-3 LC-PUFA.

The higher BF\%, insulin, HOMA-IR and TAG and lower physical activity of girls, when compared with boys, have also been described in a cohort of 386 Danish 9-year-olds ${ }^{\text {(35). }}$. American data also show higher $\mathrm{TAG}^{(36)}$ and higher HDL-cholesterol $^{(37)}$ in girls at this age. However, in contrast with the present results, Andersen et al. ${ }^{(35)}$ found slightly higher systolic and diastolic blood pressure values in boys, when compared with girls, in their Danish cohort. Whether this sex difference is explained by the higher prevalence of overweight and obesity among girls than boys of the present study is unknown.

A strength of the present study is that it is based on a homogeneous group of school children measured under highly standardised conditions and that we were able to adjust for potential mediators and confounders including parental education. The study is limited by the relatively small sample size and, theoretically, the results could be artifacts of the multiple adjustments in the regression models. However, we find this rather unlikely since the same overall pattern was seen in the simple and fully adjusted analyses. In the present study, blood pressure was measured at only one occasion. Compared with, for example, $24 \mathrm{~h}$ repeated blood pressure measurements, we may have overestimated actual resting blood pressure values due to the 'white coat syndrome'. However, all measurements were performed under standardised conditions and $24 \mathrm{~h}$ blood pressure measurements were not logistically possible in the school setting. Finally, we had no data on the pubertal status of children. Puberty affects the MetS markers and may therefore add some unexplained variation. However, since the children covered a relatively narrow age span and most were under 10 years of age and normal weight, pubertal variation among them and the resulting potential confounding effect of puberty may be limited.

In conclusion, in a group of apparently healthy, 8-11-yearold children, $n$-3 LC-PUFA status was positively associated with plasma HDL and HDL:TAG, but also with higher BF\% and lower physical activity and with higher blood pressure in boys only. This is the third time we have observed such tendencies in Danish children. The long-term consequences and potential causality and behavioural mechanisms should be investigated further.

\section{Acknowledgements}

Thanks to Stine-Mathilde Dalskov, Rikke A. Petersen, Louise B. Sørensen, Vivie K. Pedersen, Inge Rasmussen, Vivian J. Anker, Christel D. Raagaard, Debbie Nadelmann, Birgitte Hermansen, Rikke P. Laursen and Majken E. Rasmussen for their help with data collection and all the participating children, their parents and the school management and staff for their contribution to the study. C. T. D., M. F. H, A. B.-J., K. F. M. and A. A. designed the study; C. T. D., M. F. H. and A. B.-J. conducted the study; K. D. S. and L. L. analysed the data; C. T. D. and L. L. wrote the paper. All authors read and approved the final manuscript. The authors have no conflicts of interest to declare. 


\section{References}

1. Mozaffarian D \& Wu JH (2011) Omega-3 fatty acids and cardiovascular disease: effects on risk factors, molecular pathways, and clinical events. J Am Coll Cardiol 58, 2047-2067.

2. Zimmet P, Alberti G, Kaufman F, et al. (2007) The metabolic syndrome in children and adolescents. Lancet 369, 2059-2061.

3. Eisenmann JC (2008) On the use of a continuous metabolic syndrome score in pediatric research. Cardiovasc Diabetol 7, 17 .

4. Morrison JA, Friedman LA, Wang P, et al. (2008) Metabolic syndrome in childhood predicts adult metabolic syndrome and type 2 diabetes mellitus 25 to 30 years later. J Pediatr 152, 201-206.

5. Duncan GE, Li SM \& Zhou XH (2004) Prevalence and trends of a metabolic syndrome phenotype among U.S. adolescents, 1999-2000. Diabetes Care 27, 2438-2443.

6. Damsgaard CT, Schack-Nielsen L, Michaelsen KF, et al. (2006) Fish oil affects blood pressure and the plasma lipid profile in healthy Danish infants. J Nutr 136, 94-99.

7. Pedersen MH, Molgaard C, Hellgren LI, et al. (2010) Effects of fish oil supplementation on markers of the metabolic syndrome. J Pediatr 157, 395-400.

8. Campbell F, Dickinson HO, Critchley JA, et al. (2013) A systematic review of fish-oil supplements for the prevention and treatment of hypertension. Eur J Prev Cardiol 20, $107-120$

9. Lauritzen L, Harslof LB, Hellgren LI, et al. (2011) Fish intake, erythrocyte $n-3$ fatty acid status and metabolic health in Danish adolescent girls and boys. Br J Nutr 107, 697-704.

10. Asserhoj M, Nehammer S, Matthiessen J, et al. (2009) Maternal fish oil supplementation during lactation may adversely affect long-term blood pressure, energy intake, and physical activity of 7-year-old boys. J Nutr 139, 298-304.

11. Wei MY \& Jacobson TA (2011) Effects of eicosapentaenoic acid versus docosahexaenoic acid on serum lipids: a systematic review and meta-analysis. Curr Atheroscler Rep 13, 474-483.

12. Biltoft-Jensen A, Trolle E, Christensen T, et al. (2012) WebDASC: a web-based dietary assessment software for 8-11year-old Danish children. J Hum Nutr Diet (epublication ahead of print version 18 May 2012).

13. Trost SG, Loprinzi PD, Moore R, et al. (2011) Comparison of accelerometer cut points for predicting activity intensity in youth. Med Sci Sports Exerc 43, 1360-1368.

14. Cole TJ, Bellizzi MC, Flegal KM, et al. (2000) Establishing a standard definition for child overweight and obesity worldwide: international survey. BMJ 320, 1240-1243.

15. Armstrong JM, Metherel AH \& Stark KD (2008) Direct microwave transesterification of fingertip prick blood samples for fatty acid determinations. Lipids 43, 187-196.

16. Metherel AH, Taha AY, Izadi $\mathrm{H}$, et al. (2009) The application of ultrasound energy to increase lipid extraction throughput of solid matrix samples (flaxseed). Prostaglandins Leukot Essent Fatty Acids 81, 417-423.

17. Friedewald WT, Levy RI \& Fredrickson DS (1972) Estimation of the concentration of low-density lipoprotein cholesterol in plasma, without use of the preparative ultracentrifuge. Clin Chem 18, 499-502.

18. Cutfield WS, Jefferies CA, Jackson WE, et al. (2003) Evaluation of HOMA and QUICKI as measures of insulin sensitivity in prepubertal children. Pediatr Diabetes 4, 119-125.

19. Hooper L, Thompson R, Harrison R, et al. (2004) Omega 3 fatty acids for prevention and treatment of cardiovascular disease. Cochrane Database of Systematic Reviews, issue 4 CD003177.

20. Davidson MH (2006) Mechanisms for the hypotriglyceridemic effect of marine omega-3 fatty acids. Am J Cardiol 98, $27 i-33 i$.

21. Forsyth JS, Willatts P, Agostoni C, et al. (2003) Long chain polyunsaturated fatty acid supplementation in infant formula and blood pressure in later childhood: follow up of a randomised controlled trial. BMJ 326, 953-957.

22. Drew Rockett B, Harris M \& Raza Shaikh S (2012) High dose of an $n-3$ polyunsaturated fatty acid diet lowers activity of C57BL/6 mice. Prostaglandins Leukot Essent Fatty Acids 86, $137-140$.

23. Tu W, Eckert GJ, DiMeglio LA, et al. (2011) Intensified effect of adiposity on blood pressure in overweight and obese children. Hypertension 58, 818-824.

24. Whelton SP, Chin A, Xin X, et al. (2002) Effect of aerobic exercise on blood pressure: a meta-analysis of randomized, controlled trials. Ann Intern Med 136, 493-503.

25. Treuth MS, Butte NF, Adolph AL, et al. (2004) A longitudinal study of fitness and activity in girls predisposed to obesity. Med Sci Sports Exerc 36, 198-204.

26. Kodas E, Galineau L, Bodard S, et al. (2004) Serotoninergic neurotransmission is affected by $n-3$ polyunsaturated fatty acids in the rat. $J$ Neurochem $\mathbf{8 9}, 695-702$.

27. Takeuchi T, Fukumoto Y \& Harada E (2002) Influence of a dietary $n-3$ fatty acid deficiency on the cerebral catecholamine contents, EEG and learning ability in rat. Behav Brain Res 131, 193-203.

28. Hamazaki T, Sawazaki S, Itomura M, et al. (1996) The effect of docosahexaenoic acid on aggression in young adults. A placebo-controlled double-blind study. J Clin Invest $\mathbf{9 7}$, 1129-1133.

29. Buydens-Branchey L \& Branchey M (2006) n-3 Polyunsaturated fatty acids decrease anxiety feelings in a population of substance abusers. J Clin Psychopharmacol 26, 661-665.

30. Nordic Council of Ministers (2004) Nordic Nutrition Recommendations 2004. Copenhagen: Norden.

31. National Food Institute (2010) Danskernes Kostvaner 2003-2008. Hovedresultater (Dietary Habits in Denmark 2003-2008. Main Results). Søborg: Rosendahls - Schultz Grafisk A/S

32. Metherel AH, Armstrong JM, Patterson AC, et al. (2009) Assessment of blood measures of $n-3$ polyunsaturated fatty acids with acute fish oil supplementation and washout in men and women. Prostaglandins Leukot Essent Fatty Acids 81, 23-29.

33. Mozaffarian D \& Wu JH (2012) (n-3) Fatty acids and cardiovascular health: are effects of EPA and DHA shared or complementary? J Nutr 142, 614S-625S.

34. Burdge GC, Sala-Vila A, West AL, et al. (2007) The effect of altering the $20: 5 n-3$ and $22: 6 n-3$ content of a meal on the postprandial incorporation of $n-3$ polyunsaturated fatty acids into plasma triacylglycerol and non-esterified fatty acids in humans. Prostaglandins Leukot Essent Fatty Acids 77, 59-65.

35. Andersen LB, Harro M, Sardinha LB, et al. (2006) Physical activity and clustered cardiovascular risk in children: a cross-sectional study (The European Youth Heart Study). Lancet 368, 299-304.

36. Cooper GR \& Bayse DD (1985) Standardization of lipid and lipoprotein determinations for pediatric screening procedures. Prog Clin Biol Res 188, 1-17.

37. Olson RE (2000) Atherogenesis in children: implications for the prevention of atherosclerosis. Adv Pediatr 47, 55-78. 